\title{
USING PASSIVE CAVITIES FOR BUNCH SHORTENING IN CESR*
}

\author{
S. Belomestnykh ${ }^{\dagger}$, R. Kaplan, J. Reilly, J. Sikora, and V. Veshcherevich \\ Laboratory for Elementary-Particle Physics, Cornell University, Ithaca, NY 14853, USA
}

\begin{abstract}
Passive (beam-driven) superconducting cavities can be used in storage rings for bunch shortening when necessary high RF voltage can be achieved only by using multiple cavities, but the beam power consumption does not justify using all of them in the active mode, powered by klystrons. An example is the $e^{+} e^{-}$collider CESR running with a beam energy below $2.5 \mathrm{GeV}$ as a charm-tau factory (CESR-c) [1]. A short bunch length of about $10 \mathrm{~mm}$ is required for obtaining higher luminosity, while maximum beam power is only $160 \mathrm{~kW}$. Theoretical and experimental studies are in progress at CESR to investigate the collider performance at low energy in preparation for its conversion to CESR-c. In the course of these studies we looked at possible impacts of using passive cavities on the accelerator performance. The results are presented.
\end{abstract}

\section{MOTIVATION FOR USING PASSIVE CAVITIES}

CESR [1] is a single-ring $e^{+} e^{-}$collider operating in the energy range from $J / \Psi(1.55 \mathrm{GeV})$ through $Y$ resonances $(\mathrm{E} \approx 6 \mathrm{GeV})$. In the past CESR has operated mainly at $5.3 \mathrm{GeV}$ and achieved peak luminosity well above $10^{33}$ $\mathrm{cm}^{-2} \mathrm{~s}^{-1}$. Its operation range was extended recently to lower energies of charm/tau region. The low energy mode of operation, called CESR-c, utilizes short bunches and high synchrotron frequency and requires high total RF voltage (see Table 1). By raising operating gradient on some of the existing cavities and replacing others with new/refurbished cryomodules we will attain the RF voltage increase [2].

However, while the required RF voltage is high, the beam power demand is very modest and does not justify using three transmitters as in the present RF system configuration. Even one klystron is more than adequate to supply necessary power. To significantly reduce RF system power consumption and to ease stability requirements to RF controls we had proposed operating some of CESR superconducting cavities in a passive mode. A proof-of-principle experiment was performed to check feasibility of this mode of operation [3]. Measured dependence of the synchrotron frequency on the beam current was in good agreement with calculations. In this paper we present further studies of passive cavity operation in CESR-c including first experimental highenergy physics (HEP) run results.

\footnotetext{
*Work supported by the National Science Foundation. †sab@lepp.cornell.edu
}

Table 1: Selected parameters of CESR-c

\begin{tabular}{|l|c|c|c|}
\hline Energy [GeV] & 1.55 & 1.88 & 2.5 \\
\hline No. of cavities & 4 & 4 & 4 \\
Gradient [MV/m] & 6.25 & 8.33 & 10 \\
Voltage [MV] & 7.5 & 10 & 12 \\
Beam power [kW] & 40 & 90 & 160 \\
Beam current [A] & 0.26 & 0.36 & 0.46 \\
Synch. frequency [kHz] & 41 & 43 & 41 \\
Bunch length [mm] & 9.9 & 10.2 & 10.2 \\
\hline
\end{tabular}

\section{RF SYSTEM CONFIGURATION AND PARAMETERS FOR CESR-C}

CESR RF system consists of four single-cell superconducting cavity cryomodules. The cryomodules are installed in pairs in the East (E1 and E2 locations) and in the West (W1 and W2) RF straight sections of CESR. Two East cavities have individual klystrons while two West cavities share RF power from one klystron (see Figure 1).
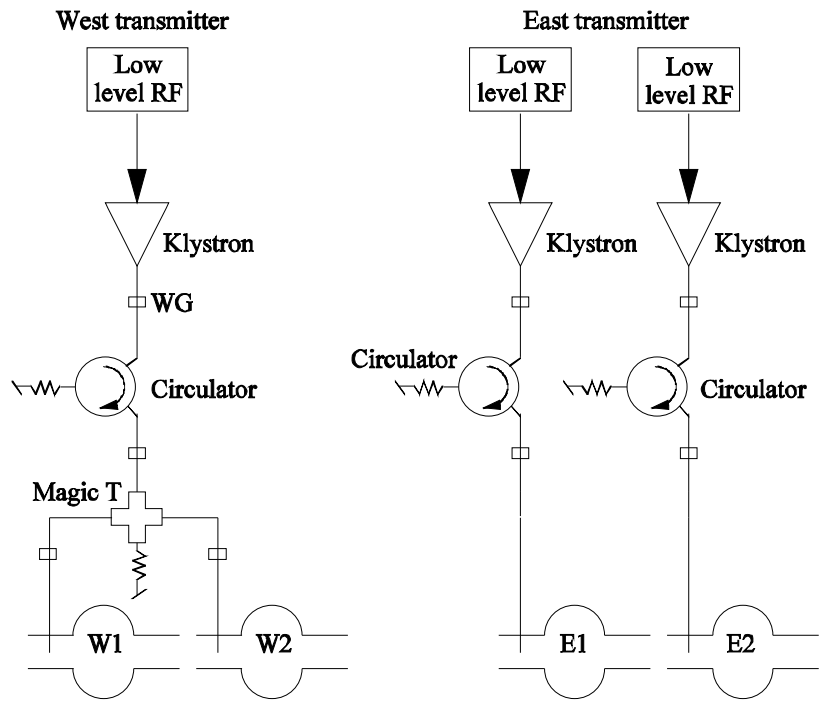

Figure 1: RF system configuration.

The proposed scheme [2] is to operate two West cavities in an active mode and two East cavities in the passive mode with the external $Q$ factor in the range between $1 \times 10^{6}$ and $3 \times 10^{6}$. We will retain klystrons connected to the passive cavities for RF processing purposes and for CESR operation at high energy for synchrotron radiation user facility. 
Requirements to the CESR-c RF system were analyzed elsewhere [2, 4]. Table 2 compares RF parameters for different number of active and passive cavities and different cavity coupling at $1.88 \mathrm{GeV}$. First set of parameters is for the configuration with four active cavities with $Q_{\text {ext }}=2.0 \times 10^{5}$, the same as we have at present. Most efficiently RF system is operating though when matched conditions are reached at the maximum beam current (second set). For CESR-c this means operating at high values of $Q_{\text {ext }}$, which will significantly increase the beam loading parameter $Y$. In the past CESR typically operated with $Y \approx 9$, which is considered heavy beam loading [4]. Using two passive cavities will alleviate the beam loading problem as well as significantly reduce power consumption (third and forth sets of parameters in Table 2).

Table 2: Comparison of RF parameters at $1.88 \mathrm{GeV}$

\begin{tabular}{|l|c|c|c|c|}
\hline $\begin{array}{l}\text { No. of active }+ \\
\text { passive cavities }\end{array}$ & $4+0$ & $4+0$ & $2+2$ & $2+2$ \\
\hline No. of klystrons & 3 & 3 & 1 & 1 \\
$Q_{\text {ext }}$ active & $2.0 \times 10^{5}$ & $3.1 \times 10^{6}$ & $6.1 \times 10^{5}$ & $1.0 \times 10^{6}$ \\
$Q_{\text {ext }}$ passive & - & - & $1.0 \times 10^{6}$ & $3.0 \times 10^{6}$ \\
$Y=V_{\mathrm{br}} / V_{\mathrm{c}}$ & 2.6 & 40.0 & 7.8 & 13.2 \\
Synchr. phase & 83.7 & 88.6 & 86.3 & 87.8 \\
$P_{\mathrm{RF}}[\mathrm{kW}]$ & 398 & 90 & 230 & 137 \\
$P_{\mathrm{AC}}[\mathrm{kW}]$ & 1150 & 810 & 470 & 370 \\
\hline
\end{tabular}

The beam acts as a transmission line between the source of RF power (active cavity) and the load (passive cavity). At a constant passive cavity voltage the power transmitted via beam stays constant. Hence the voltage seen by the beam as it passes the beam-driven cavity will depend on the beam current and will be maximal at the lowest beam current (threshold current, see next section). For example, for $1.88 \mathrm{GeV}$ conditions the voltage seen by the beam would reach $1.4 \mathrm{MV}$ at the beam current of 50 $\mathrm{mA}$, cavity voltage of $2.5 \mathrm{MV}$ and passive cavity $Q_{\mathrm{ext}}=$ $1.0 \times 10^{6}$. Energy kick due to this high voltage can create orbit perturbation (dispersion function is non-zero at the cavities' location in CESR) that may worsen machine performance. This was the reason to include two sets of parameters for passive cavities. While operating at $Q_{\text {ext }}=$ $1.0 \times 10^{6}$ is better because of the smaller beam loading, higher external $Q$ reduces the energy kick. One can improve beam loading in the latter case by operating active cavities at lower than optimal $Q_{\text {ext }}$.

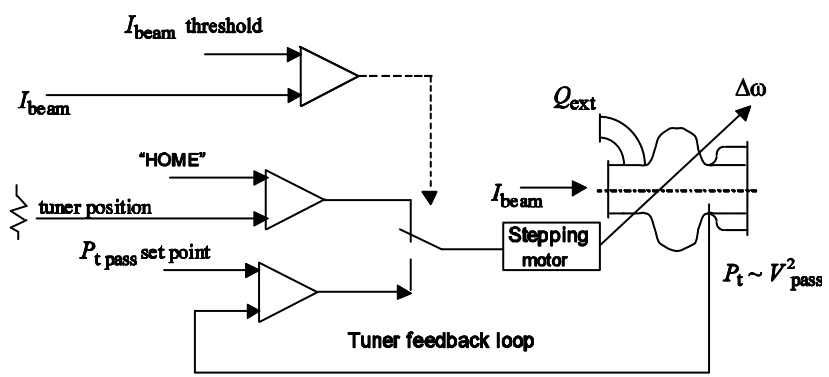

Figure 2: Passive cavity block diagram.

\section{PASSIVE CAVITY SETUP}

RF control electronics of the E2 cavity has been modified to allow operation in the passive cavity mode. Figure 2 presents the block diagram for this regime. Initially, when the beam current is below the pre-set threshold, the cavity is parked in a "HOME" position off resonance. As soon as the beam current exceeds the threshold, the tuner feedback loop is turned on and tunes the cavity frequency to keep the beam-induced voltage equal to its set point. The set-up works only on one side of the cavity resonance. One needs to change the sign of the loop gain to operate on the other side. For bunch shortening the cavity resonance frequency must be below the operating harmonic of revolution frequency. The cavity voltage set point cannot exceed $I_{\text {beam }} R / Q \cdot Q_{\text {ext }}$.

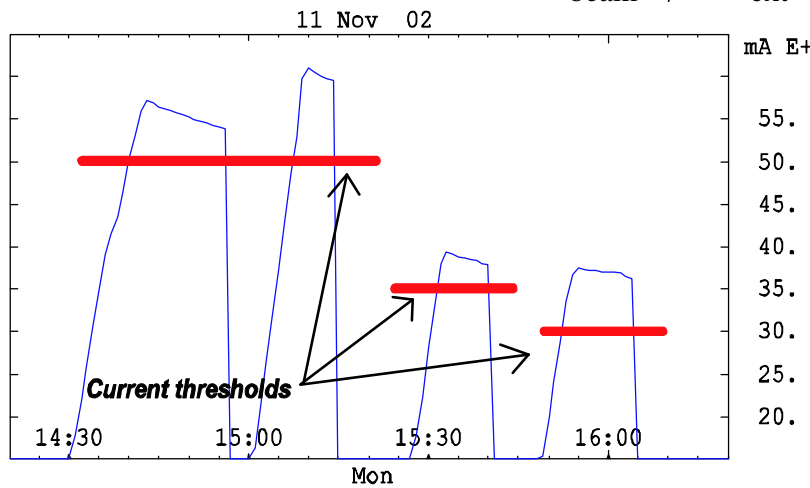

Figure 3: Beam current during first experiment.

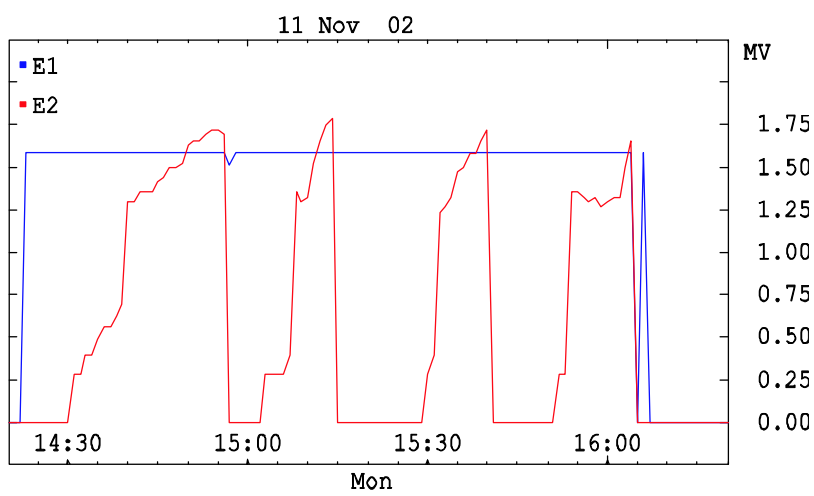

Figure 4: RF voltages during first experiment.

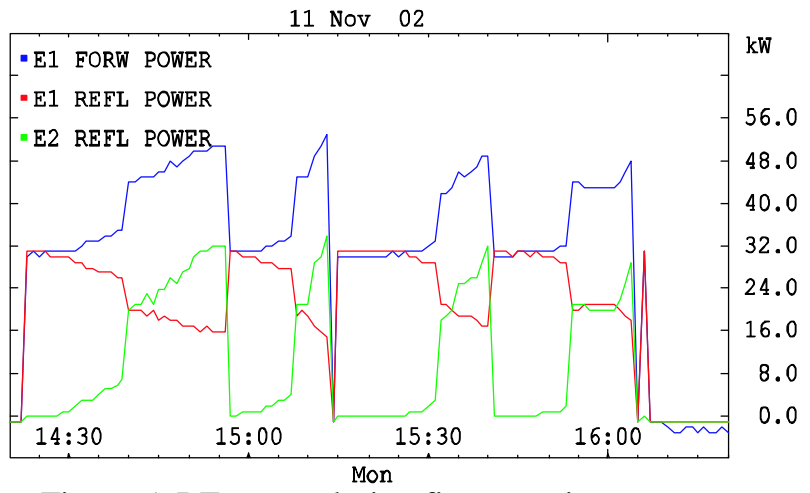

Figure 5: RF power during first experiment.
$\mathrm{kW}$

56.0

40.0

32.0

24.0

16.0

8.0

0.0 


\section{EXPERIMENTAL RESULTS}

Passive cavity experiments were performed when CESR was operating at the beam energy of $1.84 \mathrm{GeV}$ using only two cavities. The first experiment was to check operability of the passive cavity setup. E2 cavity has been switched to passive mode and its external $Q$ was adjusted to $1 \times 10^{6}$. E1 cavity remained active at $1.59 \mathrm{MV}$, West transmitter was not used at that time and both W1 and $\mathrm{W} 2$ cavities were detuned and parked off resonance. We injected positron beam to a current level slightly above the pre-set threshold and observed feedback loop operation. Then we slowly raised passive cavity field set point until RF trip due to passive cavity vacuum (Figures 3 and 4). This was repeated several times. In all cases RF tripped as soon as E2 cavity voltage reached approximately $1.9 \mathrm{MV}$. We have concluded that the trips were associated with the cavity quench and the cavity would have to be processed to operate at this level. Figure 5 illustrates how the passive cavity is loading the active one via beam current. One can see that difference between forward and reflected power on E1 cavity matches reflected power on E2 cavity.

During the second experiment RF system was switched again to one passive and one active cavity to check how passive cavity would affect luminosity during high-energy physics run. E1 (active) cavity voltage was set to 1.75 MV, E2 (passive) cavity voltage was set initially to 1.55 $\mathrm{MV}$ at first and later in the run was changed to $1.3 \mathrm{MV}$. The beam current threshold was set to a total beam current of $30 \mathrm{~mA}$. History plots of the beam currents and passive cavity voltage are shown in Figures 6 and 7 correspondingly. The luminosity was quite respectable (in comparison with normal RF setup of two active cavities) while the passive cavity voltage was set to $1.55 \mathrm{MV}$ though it degraded somewhat when the voltage was lowered. Figure 8 presents comparison of luminosity obtained with passive cavity with luminosity during one of the normal HEP runs.

\section{SUMMARY}

Experimental results obtained with one passive and one active cavity in CESR-c conditions confirm feasibility of using such a scheme. The plan is to use two passive cavities in CESR-c for more efficient running of the RF system. More experiments will be performed to study passive cavity operation at higher voltages and effects of passive cavities on beam dynamics. We hope to switch to a routine operation with two passive cavities later this year.

\section{REFERENCES}

[1] D. Rice, "CESR-c - a Frontier Machine for QCD and Weak Decay Physics in the Charm Region," Proc. of the 8th EPAC, pp. 428-430 (2002).

[2] S. Belomestnykh, "Requirements to the CESR-c RF system," Cornell LEPP Report SRF020918-06 (2002).
[3] S. Belomestnykh, et al., "Superconducting RF System Upgrade for Short Bunch Operation of CESR," Proceedings of the PAC'01, pp. 1062-1064.

[4] S. Belomestnykh, et al., "Superconducting RF Control Issues at CESR," Proceedings of the PAC'01, pp. 1065-1067.

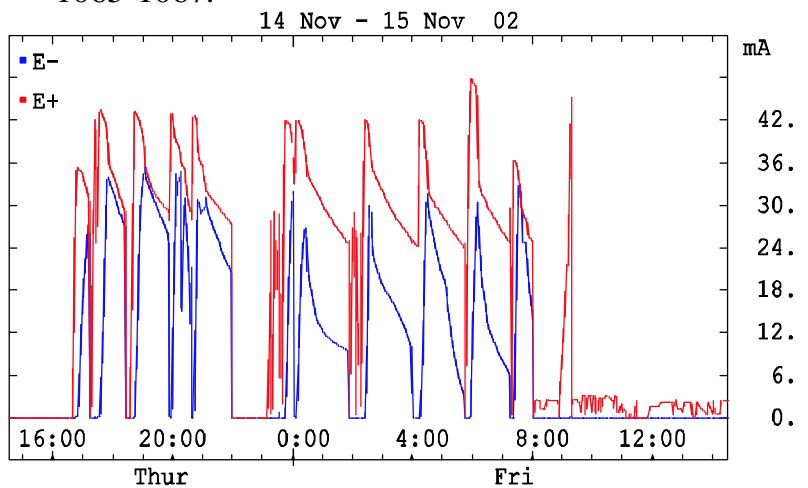

Figure 6: Beam currents during HEP run.

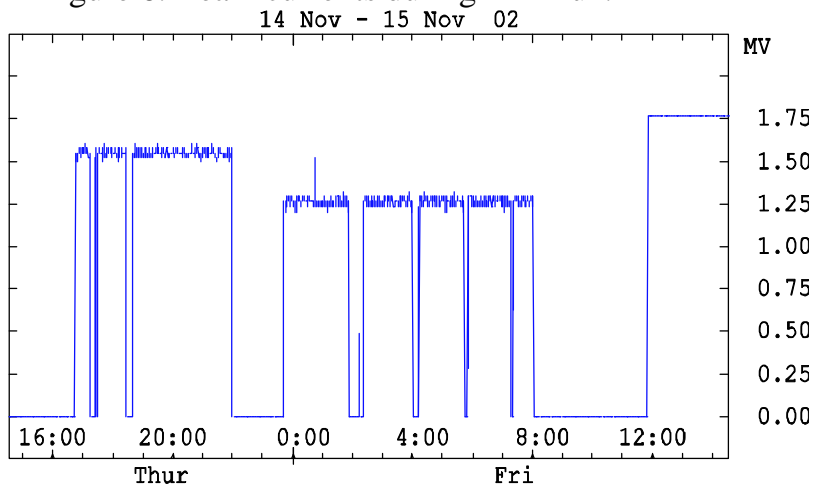

Figure 7: Passive cavity voltage during HEP run.

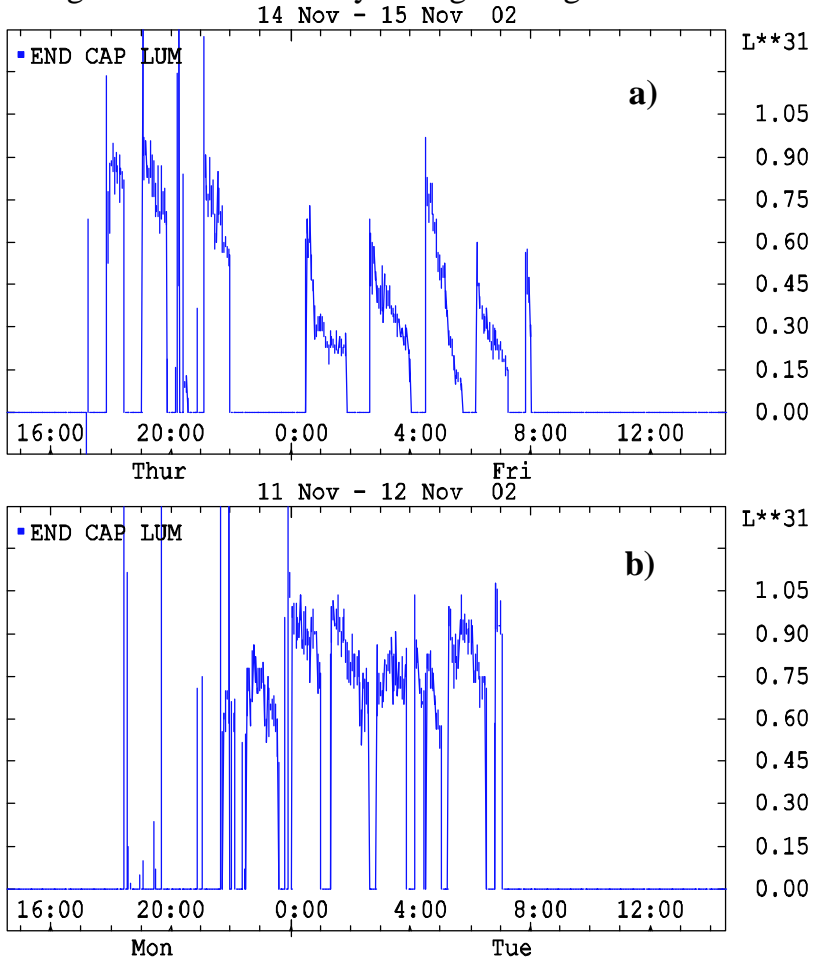

Figure 8: Luminosity during passive cavity experiment (a) and during one of the normal HEP runs (b). 